\title{
Philosophy, Education, and the Explosion of Knowledge
}

\author{
WIM J. VAN DER STEEN \\ Free University \\ and \\ PETER B. SLOEP \\ Open University
}

\begin{abstract}
In science, and in the teaching of science, knowing how is as important as knowing that. The value of theoretical knowledge is limited, especially so in view of the rapid increase in available information. Methodological analysis of concrete problems should play a major role in teaching. Also, students should learn to contextualize problems in disciplines other than their major area of interest. Generalists are badly needed for this, in teaching and in research. However, current forms of interdisciplinary integration must be de-emphasized.
\end{abstract}

KEYwORDS: philosophy, methodology, specialization, multidisciplinarity

If one describes the aims of educational programs in terms of knowledge, one should distinguish between theoretical knowledge and practical knowledge between knowing that and knowing how. Thus one can know that the structural formula for water is $\mathrm{H}_{2} \mathrm{O}$ whereas one knows how to play the piano. Accordingly, one might be led to think that in science the emphasis needs to be on knowing that, in the arts on knowing how. That conclusion, however, is insufficient. The process of generating scientific knowledge, like the process of generating works of art, calls for an apprenticeship that defies full codification. In either case it is crucially important to know existent theories and rules, and to know how to apply them.

This consideration has obvious consequences for the way one should teach science to students who want to become researchers. The transmission of theoretical knowledge (scientific or methodological) will not suffice for them. Also, those who want to come to grips with existing theoretical knowledge will need elusive ways of learning which one cannot fully specify. We will present a concrete example of this which, at the same time, will set the stage for a philosophical appraisal of current science in relation to education. Specifically, we will address the question how teachers and students should deal with steadily increasing amounts of knowledge in the life sciences. 


\section{An Example}

The example we will consider in this paper - schizophrenia - is representative of situations we encounter in teaching philosophy to students of biology. Before presenting it we have two caveats. First, the example was chosen merely because it illustrates so well the points we want to make. Perhaps some readers will feel that it is atypical since psychiatry is a problematic discipline. Concerning the points we will make, our experience with teaching in the life sciences assures us that this is not so. Second, we will concentrate on teaching at the university level; we believe, however, that our analysis is also applicable to the high school level.

A group of ten students participated in a philosophy of biology course offered by W. J. van der Steen in three-hour weekly sessions. The course was designed to train students in using philosophical tools to analyse literature in the life sciences. As undergraduates, all of the students had done an obligatory course in philosophy in the form of elementary methodology applied to biology (Van der Steen, 1982). Halfway through the course they had been asked to read a review text on schizophrenia (Schwartz \& Africa, 1984) for one of the sessions. Specifically they had been given the following task:

In the article you will find lots of details concerning the etiology and the treatment of schizophrenia. This time I suggest we won't analyze the text in detail. Instead I want you to concentrate only on the basic categories which the authors distinguish in presenting a classification of etiological factors (and other relevant factors). I would like you to comment on the classification. Application of elementary methodology you have learned will be helpful.

The classification presented by Schwartz and Africa, after deletion of descriptions, is as follows:

\section{A. Biological factors}

1. Genetic factors

2. Specific abnormalities

a. Anatomical and physiological factors

b. Biochemical factors

B. Psychosocial factors

1. Development of the individual

2. Development within the family

3. Development within society and the larger environment

a. Population density

b. Socio-economic class

c. Date of birth

d. Other factors

The set-up of the session was as follows. First, an essay written by one of the students was discussed. Second, another student gave an oral presentation 
which was followed by a discussion. Third, a plenary discussion was held focussing on the classification, which was reproduced on the blackboard. Many interesting comments were offered by the students, but no one thought at first that the classification given by the authors might be inadequate in a fundamental way. In the plenary discussion one of the students remarked that one should evaluate the classification with respect to exhaustiveness.

"Right you are," I (WJS) said (pleased to notice that an item had been picked from the textbook); "well, what about it?"

The ensuing exchange of ideas led the students to the opinion that the classification was anything but exhaustive, but they failed to argue this in a convincing way. So they had to be told. They were quite surprised that they had overlooked the obvious.

Of course the classification is exhaustive only on the assumption that the category of non-biological factors is identical with the category of psychosocial ones. At first sight the assumption is reasonable. True, one could also distinguish physical and chemical factors, but such factors may be included in the category of biological ones. That is, the authors can be taken as construing this category as a broad one. In other respects, though, they appear to have an unduly narrow view of biology. The way they have subdivided the two major categories implies that biological factors may be equated with internal factors whereas psychosocial factors are the external ones. Thus they are conflating two different dichotomies, with the result that external biological as well as internal psychosocial factors are left out.

Now one could argue that biology should suffice to characterize internal factors, so that there is no need for an additional class of internal psychosocial ones. (We would not agree, but let's take this for granted.) However, the assumption that the class of external biological factors is empty is totally indefensible. It is indeed quite plausible that ecological factors such as food or extreme physical conditions could affect one's chances to develop a psychiatric disorder. So we are dealing here with a classification in which a fundamental category is missing.

It is easy to overlook this since the basic distinction of biological and psychosocial factors is seemingly adequate. The point one should recognize is that terms one normally takes for granted, because of their apparent clarity, possibly have idiosyncratic meanings in specific contexts. In the present case, one should realize that in some areas of medicine "biology" has a much narrower meaning than the one it has for the biologist.

Incidentally, one should know that classifications like the one presented by Schwartz and Africa are common in psychiatry and various other areas of medicine, although specialized research has shown that ecological factors may be important (Van der Steen \& Thung, 1988). The implications for medicine might well be serious. 


\section{How to Teach Philosophy}

The example involves the application of theoretical knowledge at two levels, science and methodology. The point of the exercise was to give the students practical knowledge of the application of methodology to existing science. Now scientific knowledge is seldom available in a perspicuous form, but let us pretend for a while that it is not a problem. Suppose we have a lucid scientific article and an unproblematic list of methodological principles that scientific work should satisfy. Even on these assumptions, the example shows that the application of methodology to science is anything but easy. In our experience, students need to do realistic exercises over a prolonged period in which they are continually shown, retrospectively, that their answers can be improved upon without the introduction of additional theoretical knowledge.

So practical knowledge about how to apply methodological ideas to real science matters a great deal. This of course does not mean that one can dispense with theoretical (methodological) knowledge. Indeed, we feel that much can be done in the approach to teaching theoretical knowledge that would facilitate its application. In our view, philosophers in their writings tend to focus on themes which are primarily philosophically interesting. Such themes will predominantly centre on controversies in philosophy. Now methodological tools which are controversial are not easily applied in science, certainly not by scientists themselves.

Elsewhere (Sloep \& Van der Steen, 1988; Van der Steen, 1990b) we have defended the thesis that, in order to remedy this situation, one needs a different style of philosophical research, closer to that of science. Most scientists realize that it is futile to aim at pervasively valid scientific theories. Philosophers, however, wrongly believe that it is possible to aim at pervasively valid philosophical theories. We have argued, for example, that the famous deductivenomological model of explanation (Hempel \& Oppenheim, 1948) is useful even though many counter-examples show that it is not pervasively valid. Our reaction to these counter-examples is that the model should be used for particular purposes and in particular contexts (although we readily admit that Hempel and Oppenheim meant otherwise). Hempel and Oppenheim's basic intuition that explanations are arguments can be put to advantage in evaluating real science. Scientists do argue when they are giving explanations, and it makes sense to reconstruct and assess their arguments.

An important issue in more recent philosophical writings on scientific explanation (see Salmon, 1989, for a survey) is the increasing emphasis on pragmatics. The general idea .s that explanations are sensitive to context. People can ask very different questions about a phenomenon, and the adequacy of explanations given will depend on the question asked. Now philosophers who criticize Hempel and Oppenheim's model because it disregards pragmatics have got a point, but we would not conclude that the model is inadequate for this reason. We would turn the criticism on its head by extending the idea of context dependence to philosophy itself. Of course Hempel and Oppenheim's model does not 
cover all aspects of explanation; no model ever does. One should use it only in appropriate contexts in which conditions of application are satisfied. Some of the conditions can be specified (known counter-examples are useful for this purpose), for the rest one will have to be content with an open-ended ceteris paribus clause. Such clauses are part and parcel of science. Why should philosophy be different?

One could object that this line of reasoning is simplistic for the following reason. Even if all philosophers of science would heartily agree, nothing much would be gained. There are sundry models of scientific explanation. Should one accept them all with the proviso that each must be used in the proper context? If one would do that, scientists would still be left with a bewildering array of methodological tools they cannot easily apply to their work. So something more needs to be done.

As a matter of principle, this seems to be a valid objection. In practice, we think, it is not. If one's touchstone is profitability for real science, many philosophical models will turn out to be inadequate. Many of them, for example, are so complex that scientists cannot work with them. The prime issue, anyhow, is what tools are most urgently needed by the scientist. As far as the life sciences are concerned our option would be various forms of elementary logic and conceptual analysis which are relatively unproblematic (cf. our example). At this stage one might grant us this point but object that logic is not philosophy and that scientists themselves rather than philosophers should deal with problems of logic in science.

We do not agree for two reasons. First, though elementary logic is not philosophy, its application to real science does carry real philosophy in its wake. Second, live science is normally presented in succinct ways. Logic is one of the tools one needs to understand how it is structured. Philosophers who do not care for elementary reconstructions of live science will end up with a very biased view of science. They will have a preference for textbooks and theories which are well-articulated since they were accepted long ago. As a result, philosophical theories of science often do not have much of a foothold in science.

Admittedly, our line of reasoning has been programmatic. However, we will not elaborate philosophical arguments any further. Instead we will continue with the example because it illustrates yet another point we want to make: elementary methodology should be part and parcel of the teaching of science itself.

\section{The Example Continued}

The next session in the student course was again devoted to the article by Schwartz and Africa. This time the students were asked to analyse one passage in detail. Beforehand, they had been thoroughly briefed about the concept of genetic determination, which plays a central role in the passage. The text they had to analyse is reproduced below:

Genetic studies of schizophrenia have focused on the elements of consanguinity, twins, and adoption. Consanguinity studies have been the most 
frequently employed; these compare the incidence of schizophrenia in the relatives of an index case with the incidence in control families... Results indicate that closer consanguinity correlates with a higher incidence of schizophrenia....

Since it was recognized that biologic families ... also usually provided the parenting during development, the two variables of genetics and nurturing were isolated by studying children who had been adopted shortly after birth and who had subsequently developed schizophrenia. In this way, it was hoped that the environmental effects of nurturance could be separated from the genetic effects of nature. These studies confirmed the existence of a genetic component in the predisposition to schizophrenia. Nine percent of the biologic family members of schizophrenic children were themselves schizophrenic, whereas only two percent of the biologic family members of non-schizophrenic adopted children were schizophrenic; the incidence of schizophrenia in the adopting families of these two groups of children was the same.... (Schwartz \& Africa, 1984, p. 318)

The text seems to indicate that genetic factors are among the causes of schizophrenia. Before reporting the discussion during the session, let us summarize what information concerning genetic determination the students were given beforehand.

The concepts of genetic determination and environmental determination are tricky. One should realize that all features of organisms are determined by genetic and environmental factors. Strictly speaking, therefore, the statement that a particular feature of some individual organism is genetically determined is not meaningful. So it makes no sense to ask if it is true or false. It does make sense to say that differences between (or variation among) organisms in some feature are genetically determined. A statement to that effect means that a genetic difference is responsible for the difference in the feature. Notice that a difference between an organism $A$ and an organism $B$ can be genetically determined, whereas a similar difference between $A$ and $C$ is not. In rare cases, one can come across strong forms of genetic determination which warrant the assertion that a trait of an individual is genetically determined. The assertion then means that differences between the individual and any individual without the trait are due to a genetic difference.

During the discussion, the underlying argument in the text was at first reconstructed in the following way. At issue is the role of environmental factors (not to be equated with psychosocial ones) and genetic factors in the etiology of schizophrenia. If one wants to know whether genetic factors play a role at all, one needs comparisons in which the role of environmental factors can be excluded on reasonable grounds. In the investigation surveyed in the text, this was realized by studying a subpopulation consisting of persons adopted after birth. There are reasonable arguments for the conclusion that a genetic difference is among the causes of differences in schizophrenia within the subpopulation. However, the arguments are based on the assumption that there are no relevant environ- 
mental differences between schizophrenics and non-schizophrenics in the subpopulation. (The students rightly mentioned various possibilities showing that the assumption may well be incorrect, but we will not discuss this issue.) Moreover, one should be cautious with the result. Specifically, one should not simply conclude that schizophrenia in persons outside the subpopulation has a genetic cause.

Notice that this reconstruction, which does clarify a couple of things, still contains problematic phrases. "If one wants to know whether genetic factors play a role at all ..." That is an inadequate expression, since genetic factors always play a role. In the context, though, the expression may be harmless. The last sentence of the reconstruction ("Specifically ... cause") contains a more serious flaw. Does schizophrenia in persons outside the subpopulation have a genetic cause? The temptation to give a positive answer is great, but one should realize that no answer should be given at all because the question is wrong. Beyond the trivial thesis that genetic factors always play a role, nothing sensible can be said about the genetic determination of features of individuals. Instead one should make comparisons. In this case, the issue of comparisons has substantial consequences. Researchers would like to generalize findings concerning the subpopulation, but it is not at all clear how this can be done. What additional groups should one compare? There are numerous possibilities. The chances are that the role attributed to genetic factors in schizophrenia will turn out to depend strongly on the nature of (sub)populations compared. Therefore, one should be very cautious with general statements concerning the genetics of schizophrenia.

During the session, the students soon understood the conceptual problem and they agreed that differences and comparisons should be at centre stage. Conceivably, of course, there could be strong genetic determination, but the review showed that this is implausible in the extreme. Despite their understanding of the problem, the students kept falling in the conceptual trap they had uncovered. Time and again, someone would say things like "But wouldn't it be possible that a genetic factor responsible for schizophrenia in the subpopulation is likewise responsible for it in the general population?" Whereupon another student would comment: "You are doing it again!"

This does not indicate that the students were being stupid. Neither does it imply that the task of achieving conceptual clarity (not an easy one, to be sure) suffices to explain the persistent confusion among the students. Background assumptions which are widespread in science, indeed in our culture, are presumably a more important factor. We will briefly review some of these assumptions in view of implications for teaching.

\section{Being a Generalist}

Background assumptions with a bearing on the example are a mixed lot. The following overview will suffice to illustrate this.

First, there is the general subject of causality. Thinking in "linear" causal terms is common in science and in daily life. Moreover, we are used to search 
for the cause of a phenomenon, or at best for a list of the causes involved. That is, we will normally single out factors we deem causally relevant in the context we are interested in. The ensuing one-sidedness is harmless as long as the context does not demand more. In the example, the context does demand more.

Second, reductionism in various forms is common in science. Hence the role of genetic factors is over-emphasized in many contexts. Modern evolutionary biology is an example (Johnston \& Gottlieb, 1990). In biological psychiatry, undue weight is nowadays given to such factors in the study of severe disorders (Van der Steen \& Thung, 1988).

Third, it is widely assumed that generality of theories is one of the most important aims of science. With respect to biology and medicine, this aim is deeply problematic (Schaffner, 1986; Van der Steen \& Kamminga, 1992).

Fourth, there is the assumption already discussed that to understand biological aspects of a disease one must look inside the organism. Discussions of etiology in psychiatry tend to be dominated by a misleading dichotomy of biological versus psychosocial factors.

Fifth, a covert assumption is that diseases represent homogeneous categories. Nothing could be further from the truth, especially so in the case of psychiatric disorders. There cannot be any "natural" classification of diseases in view of the variability that characterizes organisms. Schwartz and Africa do emphasize this in their review, but at the same time their discussion of etiology covertly presupposes homogeneity of schizophrenia as a category.

Sixth, it is assumed that mental disorders can be characterized in value-neutral terms. It is by no means obvious that this is a reasonable assumption.

This list is not exhaustive, but it will do for our purposes. Notice that the assumptions we mentioned will mutually reinforce each other in generating misleading ideas about schizophrenia (and many other phenomena in biology and medicine). So the problems our students kept grappling with should not come as a surprise.

Background assumptions may be so deeply ingrained in our ways of thinking that it is very hard to uncover bias associated with them. What consequences should this have for teaching? We would conclude that it is useful for students to get acquainted with diverse fields of science and philosophy so that they more easily recognize bias in any particular field. The getting acquainted should not be done through a comprehensive assimilation of theoretical knowledge. That is anyhow not feasible, since theoretical knowledge in science is increasing so fast that information bearing on any particular subject tends to be inexhaustible. Apart from that, such knowledge will be useless and easily forgotten unless it is implemented in a concrete context.

Therefore, knowledge of diverse fields outside the main subjects a student is studying should come second. We would favour an educational setting in which prolonged, concrete exercises concerning main subjects have a dominant place. Theoretical knowledge of primary importance can be appended to the exercises. Thus, once students have really confronted sundry problems with the concept of genetic determination in the context of psychiatry, they will more easily under- 
stand why other disciplines are relevant for the appraisal of theories in psychiatry.

The example shows that elementary methodological analysis may help to uncover background assumptions in a natural way. An analysis of the concept of genetic determination implies that the identification of causes is contextdependent, so that statements about the genetics of characters have a limited generality. This may cast doubt on philosophical assumptions concerning the generality of biological theories. Methodological criticism of classifications of etiological factors helps to uncover the implicit assumption that ecology is irrelevant for etiology. Once such assumptions are made explicit, students will be motivated to search for relevant information in other disciplines.

In many cases, one will not need detailed information. Biology provides us with a bewildering amount of information concerning physiological aspects of schizophrenia. However, if we were asked to comment on biological aspects of schizophrenia, we would not look primarily at sundry physiological details. The first thing to notice is that there is much physiological information (which one can find in the literature if one is interested) which all in all gives a biased picture of schizophrenia in view of the glaring absence of ecological approaches. To see that, one needs to have a general idea of the structure of biology, which is not difficult at all. Over and above that, one needs to know how not to overlook the obvious, which is very difficult.

Our analysis shows that it is important that students learn to be generalists to some extent. A fortiori, teachers must develop the abilities of the generalist. For obvious reasons, we would add, generalists are needed in research as well. The current emphasis on specialization in science is unfortunate. There is a tendency to counteract it by interdisciplinary (multidisciplinary, cross-disciplinary) work. However, we are not happy with the shape this kind of work is now taking in the life sciences (Van der Steen \& Thung, 1988; Van der Steen, 1990a). For example, many researchers have aimed at an integration of biology and psychology in the last decade. This has resulted in the emergence of fields such as psychoneuroendocrinology. There are at least a dozen such fields, each with a leading high-quality journal. High quality there is, within each field. But what about the whole? Instead of an integrative view of biology and psychology we are getting lots of different integrations. Integrative views of schizophrenia are a case in point. If one takes the trouble of going through recent reviews in psychiatry journals, one will discover many different integrative accounts. We feel that the whole enterprise of interdisciplinary integration is rapidly becoming self-defeating.

One reason for this is that generality is still endorsed as a major aim of science. The various integrations all have the flavour of being encompassing. One needs excursions to adjacent fields to spot limitations. We think that science had better aim for a while at local stores of knowledge and information which can be connected in various ways as the occasion demands. If such a development were to take place, so much the better for the teaching of science. 


\section{REFERENCES}

Hempel, C. G., \& Oppenheim, P. (1948). Studies in the logic of explanation. Philosophy of Science, 15, 135-175.

Johnston, T. D., \& Gottlieb, G. (1990). Neophenogenesis: A developmental theory of phenotypic evolution. Journal of Theoretical Biology, 147, 471-495.

Salmon, W. C. (1989). Four decades of scientific explanation. Minneapolis: University of Minnesota Press.

Schaffner, K. F. (1986). Exemplar reasoning about biological models and diseases: A relation between the philosophy of medicine and philosophy of science. Journal of Medicine and Philosophy, 11, 63-80.

Schwartz, S. R., \& Africa, B. (1984). Schizophrenia disorders. In H. H. Goldman (Ed.), Reviews of general psychiatry (pp. 311-327). Los Altos: Lange Medical Publications.

Sloep, P. B., \& Steen, W. J. van der (1988). A natural alliance of teaching and philosophy of science. Educational Philosophy and Theory, 20, 24-32.

Steen, W. J. van der. (1982). A practical book on methodology for biologists. Utrecht: Bohn, Scheltema and Holkema.

Steen, W. J. van der. (1990a). Interdisciplinary integration in biology? An overview. Acta Biotheoretica, 38, 23-36.

Steen, W. J. van der. (1990b). Concepts of biology, a survey of methodological principles. Journal of Theoretical Biology, 143, 383-403.

Steen, W. J. van der., \& Kamminga, H. (1992). Laws and natural history in biology. British Journal for the Philosophy of Science, 42, 445-467.

Steen, W. J. van der., \& Thung, P. J. (1988). Faces of medicine, a philosophical study. Dordrecht: Kluwer.

Address of authors:

Dr. Peter B. Sloep

Department of Natural Science

Open University

P.O. Box 2960

6401 DL Heerlen

The Netherlands

Dr. Wim van der Steen

Faculty of Biology

Free University

De Boelelaan 1087

1081 HV Amsterdam

The Netherlands 